\title{
Asthma Management in the Era of the COVID-19 Pandemic
}

\author{
Timothy Klouda $^{1} \cdot$ Advait Pillarisetti $^{2} \cdot$ Annay Xie $^{3} \cdot$ Sushil Kabra ${ }^{4} \cdot$ Naveen Saradhi $^{3,5} \cdot$ Umakanth Katwa $^{1}$
}

Received: 7 September 2021 / Accepted: 29 September 2021/Published online: 6 November 2021

c) Dr. K C Chaudhuri Foundation 2021

\begin{abstract}
Asthma is common in children and exacerbations are usually triggered by respiratory viruses. There was considerable concern about the impact of COVID-19 on children with asthma. It was expected that children with asthma would fare poorly during the pandemic. However, the reported effect of the COVID-19 pandemic on pediatric asthma including acute asthma admissions, does not appear to be significant, but this needs careful follow-up. The socioeconomic effects of the pandemic and reduced healthcare access could potentially impact on ongoing delivery of health care in chronic respiratory conditions including asthma, especially in resource-poor settings. Children with chronic asthma need to be treated as per internationally published guidelines with innovative models of disease monitoring and ongoing care during the pandemic. During the pandemic, children with acute asthma need to be managed carefully based on local guidelines and using strict infection control policies. The use of technology such as telehealth and various tools of asthma management including questionnaires and digital monitoring will play an important role in asthma management during the pandemic. Medical professionals, healthcare administrators, and governments should be sensitive to the evolving needs of the community and work closely to continue to provide services in a challenging yet unresolved pandemic.
\end{abstract}

Keywords Asthma $\cdot$ Children $\cdot$ COVID-19 $\cdot$ Pandemic $\cdot$ Socioeconomic $\cdot$ Telehealth

\section{Background}

Asthma is one of the most common chronic respiratory diseases affecting approximately 300 million people worldwide [1]. The global prevalence of asthma symptoms in children and adolescents is estimated to be 13\% [2]. Chronic poorly controlled asthma and acute severe asthma both lead to significant morbidity and mortality. The main barriers to asthma control include poor understanding of the disease mechanisms, knowledge of medications used, poor

Umakanth Katwa

umakanth.katwa@childrens.harvard.edu

1 Department of Pediatrics, Boston Children's Hospital, Harvard Medical School, Boston, MA 02115, USA

2 School of Medicine, Apollo Institute of Medical Sciences and Research, Hyderabad, India

3 School of Medicine, University of Auckland, Auckland, New Zealand

4 Department of Pediatrics, All India Institute of Medical Sciences, New Delhi, India

5 Department of Pediatric Respiratory Medicine, Starship Children's Hospital, Auckland, New Zealand adherence, lack of follow-up, and ongoing exposure to triggers such as pollution, exercise, allergens, and smoke. Treatment strategies aim to control symptoms and decrease rates of exacerbations or hospital admissions.

This paper provides a review of effect of the pandemic on asthma presentations, treatment considerations, and the effect of other direct and indirect factors in children with asthma during the COVID-19 pandemic. The lessons learnt this far and innovations in medical model which will help manage children with asthma will also be discussed.

\section{Effects of COVID-19 on Children, Respiratory Admissions, and Health Care Utilization}

The most common presentation of COVID-19 in children includes fever, cough, rhinorrhea, tachypnea, fatigue, and gastrointestinal symptoms. Patients under the age of $18 \mathrm{y}$ with SARS-CoV-2 have less severe symptoms, with a large majority being asymptomatic or mildly symptomatic [3-6]. Unusual manifestations such as multisystem inflammatory disorder in children and adolescents are described. Suggested reasons for milder disease in children possibly include decreased ACE2 
expression in respiratory epithelium, a robust innate immune response, cross protection from other respiratory infections (e.g., rhinovirus) and fewer comorbidities [4]. Concomitant viral respiratory infection can present with fever and nasal congestion and can be confused with COVID-19 infection. Hence, the diagnostic PCR testing is critical in differentiating COVID-19 from other respiratory infections [3]. More recent COVID-19 data published from one Indian hospital revealed that $56.5 \%$ of the presenting children were managed as outpatients and 59\% of those needing admission had comorbidities [7]. Overall, $42 \%$ of the children admitted as COVID positive were hypoxic with $18 \%$ having ARDS. Mortality rate was $11.4 \%$ of the admitted children and a majority of children who died were over $10 \mathrm{y}$ old, were malnourished and/or had comorbidities such as malnutrition, tuberculosis, malignancies, etc. In another multicenter retrospective study from India, a large majority of children had mild disease and moderate-tosevere disease was present in $9.7 \%$ of the presenting children. Amongst these, $44 \%$ of the children had comorbidities with a mortality of $3.2 \%$. In another study, much milder symptoms were reported. Hence, there appears to be a clear demographic difference in severity based on various factors [6].

A study from Colorado showed that the infants younger than 3 mo and older young adults were at a higher risk of admission [8]. Children with comorbidities specifically asthma, immune deficiency, obstructive sleep apnea, obesity, and diabetes had a higher risk of admission. It may well be that children with multiple comorbidities are at a higher risk of admission.

A significant reduction in hospital and emergency care admissions of children with respiratory conditions for nonCOVID reasons has been described during the COVID-19 pandemic. While some of these could be due to a reduction in seasonal respiratory infections and transmission due to reduced human movement, some of these could be genuine lack of access to transport and reduced non-COVID emergency facilities in under-resourced and low-middle income countries. The reduced number of children presenting to usually busy tertiary respiratory clinics during the pandemic is concerning [9]. In modelling studies, it has been estimated that the risk of exponential increase in TB cases even with a temporary suspension of TB services could be catastrophic. Hence, the overall effect of COVID-19 on children can be indirect and complex, and most definitely depends on social and economic factors and these need to be considered carefully [9].

\section{Asthma as a Risk Factor for Severe COVID-19}

There was an initial concern that people with asthma were more prone to severe COVID infection due to the association of asthma with viral infections and also a theoretical risk that viral aerosol deposition is more common in them. Early data from China suggested that asthma is not a risk factor for severe infection [10]. However, studies from the UK have identified a higher risk of hospital mortality from COVID-19 for patients with an underlying diagnosis of asthma [11]. To further complicate the question, asthma has also been hypothesized to be protective of severe COVID19 symptoms, as an analysis of 57 studies including over 580,000 patients found a $14 \%$ risk-reduction ratio of acquiring COVID-19 and a 13\% decrease in hospitalizations [12]. Data on the relationship between asthma and COVID-19 in pediatric patients are lacking, with one systemic review stating that there are insufficient data to determine if there is a true risk [13].

\section{Effects of COVID-19 on Acute Asthma Exacerbations}

Viral infections are one of the most common triggers for asthma exacerbations. Surprisingly, data from various countries have shown reduced acute asthma presentations during the pandemic. One study looking at the emergency department (ED) visits at a large academic center in US found that ED visits for asthma decreased $80 \%$ immediately following the government mandated stay-at-home order [14]. Similar trends were seen in developing countries [15]. The decrease was likely a result of pandemic mitigation measures, reduced exposure to triggers such as aeroallergens, seasonal respiratory viruses, air pollution and smoking. Similar trends in asthma are seen when children are removed from potential triggers and allergens during school vacations [16]. Admissions for asthma, along with other winter dominate illnesses including bronchiolitis and pneumonia have declined in 2020 [17]. It should be noted that after these restrictions are removed and start of school there is a possibility that increase in surge of viral infections which in fact can trigger asthma flare ups. Multiple cities including Queensland in Australia have demonstrated increased seasonal viral such as RSV and rhinovirus infections out of season [18].

\section{Risk Factors Associated with Asthma}

Allergic rhinitis and atopy are major risk factors for asthma and its treatment need to be optimized. Children with symptoms such as rhinorrhea, cough, pharyngitis, and nasal congestion seen from allergic rhinitis can be confused with COVID-19 symptoms and should be tested if required. Obstructive sleep apnea (OSA), a systemic inflammatory disorders due to dysfunctional breathing during sleep can worsen airway inflammation in asthmatics and has been described as a potential risk factor for severe COVID-19 
infection in adults [19]. Hence, children with obesity and asthma are at higher risk of OSA, which further increases the risk of severe COVID-19 and hence these conditions need to be evaluated and treated appropriately. During the COVID19 pandemic obesity rates have increased from $13.7 \%$ to 15.4\%, with the highest increase seen in Hispanic/Latino and non-Hispanic black patients 5 to 9 y old [20].

Sleep deprivation and fragmentation can alter immune response and can potentially increase the risk of severe COVID-19; hence, promotion of good sleep hygiene practices, in addition to management of anxiety and depression and dysfunctional breathing are all regarded as important adjuncts to asthma management. There is a well-documented association between psychosocial risk factors and poor asthma control [21]. During the pandemic, children, and adolescents with asthma have reported high rates anxiety, depression, and post-traumatic stress [22].

\section{Socioeconomic Status}

The incidence and severity of asthma is higher in low income households and minorities, with asthma being nearly twice more likely among select minority groups [23]. Children living in a low-income household have a twofold increased risk of developing asthma by age 14 [24]. COVID-19 infection rates and mortality has been higher in patients with socioeconomic risk factors, including household composition, minority status and environmental status [25]. This disparity is possibly due to limited access to medical care, household composition and occupational exposure. A comprehensive review of a patient's social history can identify barriers contributing suboptimal health care.

\section{Management of Asthma During the COVID-19 Pandemic}

Multiple organizations such as the Global Initiative for Asthma (GINA) and the Center for Disease Control (CDC) have released guidelines for asthma care in pediatric patients generally and also specifically during the COVID pandemic [26]. A metered dose inhaler (MDI) with spacer and appropriately fitting face mask/mouthpiece or a dry powder inhaler is the preferred route for inhaled medications. When possible, nebulizers should be avoided to reduce the risk of aerosolization and potential spread of SARS-CoV-2, which can infect others [27]. Spirometry, another aerosol generating procedure, should be done with proper infection control.

At every visit, either virtual or in person, asthma action plans should be provided along with education. Review of spacer and MDI technique should be performed. The use of multimedia videos or pictures can be helpful in demonstrating techniques via telemedicine. The Asthma Control Test (ACT) is a validated tool in the management of asthma, demonstrating the ability to discriminate asthma severity, predict FEV1 and indicate the need to alter controller medications [28]. These questionnaires have an important role in asthma management, as studies have shown relying on caregiver's history results in underestimating symptoms [29]. GINA guidelines, widely used in many countries recommend the use of daily low dose ICS in place of SABA as first-line therapy. Patients can also feel a false confidence in controlling symptoms with a SABA, making them less likely to seek medical care when needed. Frequent use of SABAs has also been associated with downregulation of beta-receptors and decreased bronchoprotection [30]. Use of a SMART regime in adolescents is being increasingly recommended [31].

\section{Management of Acute Asthma Exacerbations During the COVID-19 Pandemic}

There can be significant overlap between symptoms of an asthma exacerbation and COVID-19 infection. At the first sign of any illness, patients should follow their asthma action plan, and a consultation with a patient's primary care provider to determine if testing for COVID-19 is needed. Patients with mild symptoms can follow their asthma action plan for relief therapy as needed. Patients in moderate-tosevere distress or not responding to relief therapy should seek medical attention.

Children with worsening respiratory distress, poor oral intake, hypoxemia, or not improving following their asthma action plan at home should be evaluated for admission. Patients admitted should be placed on appropriate precautions including use of PPE, negative pressure room if available, have continuous monitoring along with oxygen support as needed and cautious administration of IV fluids due to risk of fluid overload and pulmonary edema. Oral corticosteroids are not routinely recommended for mild COVID-19 infection but can be considered in those with asthma exacerbation [32]. Those not improving to asthma medications and supportive care should have additional testing including chest radiographs and labs to identify other possible sources of infection and the need for antibiotics. Chest CT scans are currently not routinely recommended in pediatric patients for diagnosis or evaluation of COVID-19 [33]. Currently there are no therapies specifically approved for treatment of COVID-19 in children. Additional therapies for management of RT-PCR diagnosed COVID-19 infection in children with asthma exacerbations should be based on case-by-case basis and risk analysis and in consultation with local pediatric COVID experts to avoid unnecessary side effects. All 
patients after infection should follow local or international guidelines for quarantine, returning to school or other activities and retesting.

\section{Primary Prevention, Reducing Transmission and Vaccines}

Vaccines provide hope in preventing COVID and reducing severity of infections including recent viral variants. Hand hygiene measures, shielding of the vulnerable, isolation and quarantine, lockdowns and mask wearing have all helped with reduced viral transmission. Multiple studies have demonstrated the safety and effectiveness of masks decreasing transmission of SARS-CoV-2 and other viruses when worn properly [34].

\section{Barriers to Healthcare}

Multiple barriers to accessing medical care during the COVID-19 pandemic include lockdowns, changes in health care facility guidelines, social distancing limitations, and quarantine recommendations. These variables have directly affected patient volume, as new patient appointments and the total number of patients seen by providers was reduced. In an online survey of 91 providers who are members of the Pediatric Asthma in Real Life think tank and World Allergy Organization Pediatric Asthma Committee, 39\% ceased in person appointments, 50\% stopped taking new patients, and 75\% limited patients visits due to the COVID-19 pandemic. Despite limited access to health care, patient control was negatively affected in only $10 \%$ of the patients, and $20 \%$ of patients reported improvement better than expected and increased adherence to medications [35]. The rising poverty and unemployment may further amplify health inequalities.

\section{Virtual Clinics and Telehealth}

There was a reported reduction in the number of respiratory and asthma clinics during COVID-19 due to re-organization of medical care. Telephonic and online web-based platforms were increasingly adopted in clinics across the world with reasonable satisfaction [36, 37]. Use of validated questionaries such as the Asthma Control Test (ACT) and Asthma Control Questionnaire (ACQ) was promoted in many of these clinics to good effect. Use of peak flow meters and portable spirometry were reported in developing and developed countries. Overall questionnaire-based studies appeared to indicate good adherence to treatment and good asthma control overall in children with asthma during the pandemic.
There are opportunities to help improve the efficiency of virtual clinics by using various well-known tools of asthma management [38]. During the physical exam of a child with asthma, the evaluation of comorbidities such as allergic rhinitis, obesity, tonsillar hypertrophy, and eczema is vital. Signs such as clubbing, wheezing, hypoxemia, and increased work of breathing should be carefully evaluated. Surveys at an academic pediatric pulmonary clinic categorized the lack of physical exam as a moderate or high level of concern in about 53\% of patients [39]. Despite inherent limitations, most providers and patients who utilized telehealth platforms seem to report a positive experience [39]. However, there are privacy and confidentiality issues, and financial and medicolegal implications which need to be addressed. Telehealth clinics experience could be enhanced by good video links, integrated examination (e.g., telestethoscope), validated questionnaires, and also locally developed checklists which can help detect red flags and help evaluate other conditions which can present like asthma. Supporting medical trainees through supervision and ongoing continuing medical education by national and international pediatric and thoracic societies, teleconferences, and case discussion can open several opportunities which can increase access to global experts and enhance training of pediatric specialist.

\section{Conclusion}

While the effect of the COVID-19 pandemic on health care systems across the world has been catastrophic, children seem to be relatively spared. Children with asthma do not appear to be disproportionally affected by the COVID -19 pandemic. There is, however, a risk of reduced access to health care, treatment, and disease monitoring if the pandemic continues, especially in poorer countries and among the socially and economically disadvantaged. Adopting models of care such as telehealth, using validated questionnaires, and home-based monitoring, and where applicable, use of cloud technology can possibly change the future of managing chronic respiratory conditions including asthma for the better. These paradigm changes in patient management and innovative approaches to medical care may mitigate some of the effects of the pandemic on health care delivery and in management of asthma.

Authors' Contributions TK, AP, AX: Literature search, writing; SK, NS, UK: Conception, writing, editing; Dr. Naveen Pillarisetti, Pediatric Respiratory Medicine, Starship Hospital, Auckland, New Zealand will act as the guarantor for this paper.

\section{Declarations}

Conflict of Interest None. 


\section{References}

1. GBD 2015 Chronic Respiratory Disease Collaborators. Global, regional, and national deaths, prevalence, disability-adjusted life years, and years lived with disability for chronic obstructive pulmonary disease and asthma, 1990-2015: a systematic analysis for the global burden of disease study 2015. Lancet Respir Med. 2017;5:691-706.

2. Ferrante G, La Grutta S. The burden of pediatric asthma. Front Pediatr. 2018;6:186.

3. Ding Y, Yan H, Guo W. Clinical characteristics of children with COVID-19: a meta-analysis. Front Pediatr. 2020;8:431.

4. Zimmermann P, Curtis N. Coronavirus infections in children including COVID-19: an overview of the epidemiology, clinical features, diagnosis, treatment and prevention options in children. Pediatr Infect Dis J. 2020;39:355-68.

5. Jat KR, Sankar J, Das RR, et al. Clinical profile and risk factors for severe disease in 402 children hospitalized with SARS-CoV-2 from India: Collaborative Indian Pediatric COVID Study Group. J Trop Pediatr. 2021;67:fmab048.

6. Nallasamy K, Angurana SK, Jayashree M, et al. Pediatric COVID Management Team. Clinical profile, hospital course and outcome of children with COVID-19. Indian J Pediatr. 2021;88:979-84.

7. Singh P, Attri K, Mahto D, et al. Clinical profile of COVID-19 illness in children-experience from a tertiary care hospital. Indian J Pediatr. 2021. https://doi.org/10.1007/s12098-021-03822-5.

8. Graff K, Smith C, Silveira L, et al. Risk factors for severe COVID19 in children. Pediatr Infect Dis J. 2021;40:e137-45.

9. Lodha R, Kabra SK. COVID-19 pandemic: impact on health care of children and the urgent need to restore regular healthcare services. Indian J Pediatr. 2021;88:225-6.

10. Li X, Xu S, Yu M, et al. Risk factors for severity and mortality in adult COVID-19 inpatients in Wuhan. J Allergy Clin Immunol. 2020;146:110-8.

11. Williamson E, Walker AJ, Bhaskaran K, et al. OpenSAFELY: factors associated with COVID-19-related hospital death in the linked electronic health records of 17 million adult NHS patients. medRxiv. 2020. https://doi.org/10.1101/2020.05.06.20092999

12. Sunjaya AP, Allida SM, Di Tanna GL, Jenkins C. Asthma and risk of infection, hospitalization, ICU admission and mortality from COVID-19: systematic review and meta-analysis. J Asthma. 2021:1-14. https://doi.org/10.1080/02770903.2021.1888116.

13. Castro-Rodriguez JA, Forno E. Asthma and COVID-19 in children: a systematic review and call for data. Pediatr Pulmonol. 2020;55:2412-8.

14. Simoneau T, Greco KF, Hammond A, Nelson K, Gaffin JM. Impact of the COVID-19 pandemic on pediatric emergency department utilization for asthma. Ann Am Thorac Soc. 2020;18:717-9.

15. Alsulaiman JW, Kheirallah KA, Ajlony MJ, Al-Tamimi TM, Khasawneh RA, Al-Natour L. Paediatric asthma exacerbation admissions and stringency of non-pharmaceutical interventions: results from a developing country. Int J Clin Pract. 2021;75:e14423.

16. Hatoun J, Correa ET, Donahue SMA, Vernacchio L. Social distancing for COVID-19 and diagnoses of other infectious diseases in children. Pediatrics. 2020;146:e2020006460.

17. Pelletier JH, Rakkar J, Au AK, Fuhrman D, Clark RSB, Horvat CM. Trends in US pediatric hospital admissions in 2020 compared with the decade before the COVID-19 pandemic. JAMA Netw Open. 2021;4:e2037227.

18. Foley DA, Yeoh DK, Minney-Smith CA, et al. The interseasonal resurgence of respiratory syncytial virus in australian children following the reduction of coronavirus disease 2019-related public health measures. Clin Infect Dis. 2021. https://doi.org/10.1093/cid/ciaa1906.
19. Strausz S, Kiiskinen T, Broberg M, et al. Sleep apnoea is a risk factor for severe COVID-19. BMJ Open Respir Res. 2021;8:e000845.

20. Jenssen BP, Kelly MK, Powell M, Bouchelle Z, Mayne SL, Fiks AG. COVID-19 and changes in child obesity. Pediatrics. 2021;147:e2021050123.

21. Weinstein SM, Pugach O, Rosales G, Mosnaim GS, Walton SM, Martin MA. Family chaos and asthma control. Pediatrics. 2019;144:e20182758.

22. Marques de Miranda D, da Silva Athanasio B, Sena Oliveira AC, Simoes-E-Silva AC. How is COVID-19 pandemic impacting mental health of children and adolescents? Int J Disaster Risk Reduct. 2020;51:101845.

23. Vazzalwar N, Totapally B, Totapally M. Racial/Ethnic disparities Among Children with Asthma Discharged from the Hospital. Pediatrics. 2019. https://doi.org/10.1542/peds.144.2_MeetingAbstract. 508.

24. Kozyrskyj AL, Kendall GE, Jacoby P, Sly PD, Zubrick SR. Association between socioeconomic status and the development of asthma: analyses of income trajectories. Am J Public Health. 2010;100:540-6.

25. Karmakar M, Lantz PM, Tipirneni R. Association of social and demographic factors with COVID-19 incidence and death rates in the US. JAMA Netw Open. 2021;4:e2036462.

26. Global Initiative for Asthma. Global Strategy for Asthma Management and Prevention. 2021. Available at: www.ginaasthma.org. Accessed on 24th Aug 2021.

27. Amirav I, Newhouse MT. Transmission of coronavirus by nebulizer: a serious, underappreciated risk. CMAJ. 2020;192:E346.

28. Nathan RA, Sorkness CA, Kosinski M, et al. Development of the asthma control test: a survey for assessing asthma control. J Allergy Clin Immunol. 2004;113:59-65.

29. Davis KJ, Disantostefano R, Peden DB. Is Johnny wheezing? Parent-child agreement in the childhood asthma in america survey. Pediatr Allergy Immunol. 2011;22:31-5.

30. Hancox RJ, Cowan JO, Flannery EM, Herbison GP, McLachlan CR, Taylor DR. Bronchodilator tolerance and rebound bronchoconstriction during regular inhaled beta-agonist treatment. Respir Med. 2000;94:767-71.

31. Cloutier MM, Dixon AE, Krishnan JA, Lemanske RF Jr, Pace W, Schatz M. Managing asthma in adolescents and adults: 2020 asthma guideline update from the national asthma education and prevention Program. JAMA. 2020;324:2301-17.

32. Interim clinical guidance for management of patients with confirmed coronavirus disease (COVID-19). Centres for Disease Control and Prevention. 2021. Available at: https://www.cdc.gov/ coronavirus/2019-ncov/hcp/clinical-guidance-managementpatients.html. Accessed on 24th Aug 2021.

33. Foust AM, Phillips GS, Chu WC, et al. International expert consensus statement on chest imaging in pediatric COVID-19 patient management: imaging findings, imaging study reporting, and imaging study recommendations. Radiol Cardiothorac Imaging. 2020;2:e200214.

34. Brooks JT, Butler JC. Effectiveness of mask wearing to control community spread of SARS-CoV-2. JAMA. 2021;325:998-9.

35. Papadopoulos NG, Custovic A, Deschildre A, et al. Pediatric Asthma in Real Life Collaborators. Impact of COVID-19 on pediatric asthma: practice adjustments and disease burden. J Allergy Clin Immunol Pract. 2020;8:2592-9.

36. Kumari J, Jat KR, Kabra SK. Role of telemedicine in follow-up care of children with respiratory illnesses at a tertiary care hospital - an ambispective observational study. Indian J Pediatr. 2021;88:974-8.

37. Jain S, Thakur C, Kumar P, Goyal JP, Singh K. Telemedicine for asthma follow-up in children during COVID-19 pandemic. Indian J Pediatr. 2021;88:1050. 
38. Mulholland A, Ainsworth A, Pillarisetti N. Tools in asthma evaluation and management: when and how to use them? Indian J Pediatr. 2018;85:651-7.

39. Davis J, Gordon R, Hammond A, et al. Rapid implementation of telehealth services in a pediatric pulmonary clinic during COVID-19. Pediatrics. 2021;148:e2020030494.
Publisher's Note Springer Nature remains neutral with regard to jurisdictional claims in published maps and institutional affiliations. 